Disclosure of Interests: None declared

DOI: 10.1136/annrheumdis-2021-eular.3162

\section{POS0763 PERFORMANCE OF THE NEW ACR/EULAR 2019 CLASSIFICATION CRITERIA FOR SYSTEMIC LUPUS ERYTHEMATOSUS (SLE) IN A COHORT OF ARGENTINIAN PATIENTS}

J. M. Dapeña ${ }^{1}$, E. R. Serrano ${ }^{1}$, J. M. Bande ${ }^{1}$, M. A. Medina ${ }^{1}$, D. S. Klajn ${ }^{1}$, J. A. Caracciolo ${ }^{1}$, C. Castro ${ }^{2}$, J. Morbiducci ${ }^{2}$, A. L. Mercé2, R. Tralice ${ }^{1,2}$, G. V. Espasa ${ }^{3}$, S. Yessika Jackeline ${ }^{3}$, M. L. Leguizamón ${ }^{3}$, M. A. Pera ${ }^{3}$, V. I. Bellomio ${ }^{3}$, M. S. Yacuzzi ${ }^{4}$, M. Machado Escobar ${ }^{4}$, M. Cosentino ${ }^{5}$, L. Garcia ${ }^{5}$, M. García ${ }^{5}$, C. Aeschlimann ${ }^{6}$, G. Gomez ${ }^{7}$, N. Perez ${ }^{7}$, S. Papasidero ${ }^{1}{ }^{1}$ Hospital General de Agudos Dr. Enrique Tornú, Servicio de Reumatología, Buenos Aires, Argentina; ${ }^{2}$ Hospital General de Agudos Bernardino Rivadavia, Servicio de Reumatología, Buenos Aires, Argentina; ${ }^{3}$ Hospital Ángel C. Padilla Juan Bautista Alberdi, Servicio de Reumatología, San Miguel de Tucumán, Argentina; ${ }^{4}$ Hospital Eva Perón de Tucumán, Servicio de Reumatología, Banda del Río Sali, Argentina; ${ }^{5}$ H.I.G.A. San Martín de La Plata, Servicio de Reumatología, La Plata, Argentina; ${ }^{6}$ Hospital Provincial de Rosario, Servicio de Reumatología, BZL, Argentina; ${ }^{7}$ Instituto de Investigaciones Médicas Alfredo Lanari, Servicio de Reumatología, Buenos Aires, Argentina

Background: In 2019 ACR and EULAR published in joint collaboration the new classification criteria for Systemic Lupus Erythematosus (SLE). Compared to the previous ones, these criteria have shown higher sensitivity and specificity in multiple cohorts. To our knowledge, its performance has not been evaluated in a cohort of patients with rheumatological diseases living in Argentina.

Objectives: The aim of this study was to evaluate the sensitivity and specificity of the ACR/ EULAR 2019 criteria in a cohort of patients with connective tissue diseases residing in Argentina. Secondary objectives were to determine the Likelihood Ratio (LR) of these criteria and the correlation of their global score with activity and damage indexes of the disease.

Methods: Multicentre, retrospective and analytical study. Patients $\geq 18$ years old with diagnosis of SLE (ACR 1997/SLICC 2012) without other associated collagen diseases (case group), and patients with other non-SLE connective tissue diseases (control group) were included. Those with active infectious disease, oncohematological disease, drug-induced lupus and overlap syndrome were excluded. Sociodemographic data, characteristics of the disease and treatment were recorded. In addition, activity and damage indexes were recorded in the group with SLE.

Three SLE experts, blinded to the diagnosis determined, for every individual if the patient had SLE or another rheumatological disease. An interrater agreement of $100 \%$ (including the 3 evaluators) was considered "defined SLE" and used as gold standard. In all cases, ACR 1997/SLICC 2012/ACR / EULAR 2019 criteria were applied and compared with the gold standard. Statistical analysis: Descriptive statistics was estimated. Sensitivity, specificity, positive and negative LR of the criteria were determined. The association between the final score of the ACR-EULAR 2019 criteria and the disease activity and damage indexes were estimated with Spearman correlation test. STATA 15.0 was used for data analysis.

Results: A total of 365 patients from 7 centres in Argentina were included. A One hundred and eighty-three belonged to the SLE group: $92.3 \%$ women, mean age 39 years (SD 13.3), median disease duration 92 months (IQR 37-150). The most frequent manifestations of the disease were mucocutaneous (94\%), musculoskeletal $(82.5 \%)$ and haematological $(69 \%)$. All patients presented ANA +, $88 \%$ hypocomplementemia, $69.4 \%$ Anti-DNA and $19.5 \%$ antiphospholipid antibodies. Median SLEDAI and SLICC were 2 (IQR 0-6) and 0 (IQR 0-1), respectively. In the control group, 182 patients were recruited: $84 \%$ women, mean age 53.6 years (SD 14.2) and median disease duration 82.5 months (IQR 38-151). The most frequent diseases were Rheumatoid Arthritis (46.1\%), Scleroderma (18.1\%) and Sjögren's Syndrome (16.5\%) and most common manifestations were musculoskeletal (81.9\%), immunological $(73.6 \%)$ and constitutional (25.3\%). A total of $62.6 \%$ of patients presented ANA+, $8.6 \%$ hypocomplementemia, and $1.3 \%$ Antiphospholipid antibodies.

Ninety-one percent of patients in the case group were classified as defined SLE and $3.8 \%$ in the control group.

The ACR / EULAR 2019 Criteria showed a 99.4\% sensitivity and an $89.1 \%$ specificity, with a LR+ of 9.1 and a LR- of 0.007 . The sensitivity and specificity of SLICC 2012 criteria were $98.3 \%$ and $88 \%$, respectively with a LR+ of 8.2 and a LR- of 0.02 ; and the ACR 1997 criteria showed a $93.96 \%$ sensitivity and $90.1 \%$ specificity, with LR + of 8.21 and $L R$ - of 0.07 .

The correlations between the ACR/EULAR 2019 Criteria global score, and activity and damage indexes were 0.19 and -0.006 , respectively.

Conclusion: The new ACR / EULAR 2019 criteria have shown high sensitivity, a specificity comparable to its predecessors, and a higher ability to distinguish SLE from other diseases and to exclude it in non-SLE patients. No correlation was observed between the criteria scores and activity and damage indexes.

\section{REFERENCES:}

[1] Aringer M, Costenbader K, Daikh D, et al 2019 EULAR/ACR classification criteria for SLE. Ann Rheum 2019; 78: 1151-1159.

Disclosure of Interests: None declared

DOI: 10.1136/annrheumdis-2021-eular.3172

\section{POS0764 EULAR RECOMMENDATION-BASED QUALITY INDICATORS (QIS) FOR SYSTEMIC LUPUS ERYTHEMATOSUS (SLE): ELABORATION, FINAL SET, PERFORMANCE AND INITIAL VALIDATION}

K. Chavatza ${ }^{1}$, M. Kostopoulou' ${ }^{2}$, D. Nikolopoulos ${ }^{1}$, O. Gioti ${ }^{3}$, K. Togia $^{1}$ S. Flouda', N. Kapsala', M. Kosmetatou', G. S. Moysidou', A. Grivas ${ }^{1}$ A. Pieta ${ }^{1}$, A. Ntourou ${ }^{1}$, P. Rapsomaniki ${ }^{1}$, T. Gerogianni ${ }^{1}$, D. Tseronis ${ }^{1}$, M. Aggelakos ${ }^{1}$, T. Karageorgas ${ }^{1}$, P. Katsimpri ${ }^{1}$, L. Andreoli ${ }^{4,5}$, M. Aringer $^{6}$, J. N. Boletis ${ }^{7}$, A. Doria ${ }^{8}$, F. Houssiau ${ }^{9}$, D. Jayne ${ }^{10}$, M. Mosca ${ }^{11}$, E. Svenungsson ${ }^{12}$, A. Tincani ${ }^{13}$, G. Bertsias ${ }^{14}$, A. Fanouriakis ${ }^{1,3}$, D. Boumpas ${ }^{1,15}$. "Attikon" University Hospital of Athens, Rheumatology and Clinical Immunology, Medical School, National and Kapodistrian University of Athens, Athens, Greece; " $\mathrm{G}$. Gennimatas" General Hospital, Department of Nephrology, Athens, Greece; 3"Asklepieion" General Hospital, Voula, Department of Rheumatology, Athens, Greece: ${ }^{4}$ University of Brescia, Department of Clinical and Experimental Sciences, Brescia, Italy; ${ }^{5}$ Spedali Civili, Unit of Rheumatology and Clinical Immunology, Brescia, Italy; ${ }^{6}$ University Medical Center \& Faculty of Medicine Carl Gustav Carus, Technical University of Dresden, Division of Rheumatology, Department of Medicine III, Dresden, Germany; "“Laikon” Hospital, National and Kapodistrian University of Athens, Medical School, Nephrology Department and Renal Transplantation Unit, Athens, Greece; ${ }^{8}$ University of Padova, Rheumatology Unit, Department of Medicine, Padova, Italy; ${ }^{9}$ Université catholique de Louvain, Cliniques Universitaires Saint-Luc, Brussels, Belgium; ${ }^{10}$ Addenbrooke's Hospital, Department of Medicine, Cambridge, United Kingdom; ${ }^{11}$ University of Pisa, Rheumatology Unit, Department of Clinical and Experimental Medicine, Pisa, Italy: ${ }^{12}$ Karolinska Institutet and Karolinska University Hospital, Department of Medicine, Rheumatology Unit, Stockholm, Sweden; ${ }^{13}$ University of Brescia, Rheumatology and Clinical Immunology, Brescia, Italy; ${ }^{14}$ University Hospital of Heraklion, Rheumatology, Clinical Immunology and Allergy, Heraklion, Greece; ${ }^{15}$ Biomedical Research Foundation of the Academy of Athens, Laboratory of Autoimmunity and Inflammation, Athens, Greece

Background: Targets of therapy and quality of care are receiving increased attention in systemic lupus erythematosus (SLE).

Objectives: To develop Quality Indicators (QIs) for the care of SLE patients based on the EULAR recommendations, and assess their performance.

Methods: Using the published EULAR recommendations for SLE, we developed 44 candidate QIs. These were independently rated for validity and feasibility by 12 experts, analysed by a modified RAND/UCLA model and further scrutinized based on the scorings and expert opinion. (Fig.1) Adherence to the final set of Qls was tested in a cohort of 220 SLE patients combined with an assessment on its impact on disease outcomes such as flares, hospitalizations and organ damage.

Results: The panel rated 18 QIs as valid and feasible. These involve diagnosis; disease and damage assessment; monitoring for lupus nephritis and drug toxicity; therapy and targets of therapy; fertility and pregnancy; and adjunct therapy (preventive measures for osteoporosis, vaccination, cardiovascular disease). On average, SLE patients received $54 \%(95 \% \mathrm{Cl} 52-56 \%)$ of the indicated care with adherence ranging from $41 \%$ for Qls related to monitoring to $88 \%$ for treatment-related QIs. Regarding targets of therapy, sustained remission or low disease activity were achieved in $27 \%$, while $94 \%$ of patients received low-dose glucocorticoids, and $92 \%$ the recommended hydroxychloroquine dose. Dependent upon individual QI tested, adherence for lupus nephritis-related Qls was $88 \%$ for receiving appropriate adjunct therapy (ACE inhibitors) to $100 \%$ for being treated with the indicated immunosuppressive treatment. In contrast, adherence to Qls related to preventive measures and other adjunct therapies was moderate to low. Notably, patients who were eligible for cardiovascular risk modification, vaccination, and osteoporosis management received lower quality of care ( $40.5 \%, 47.7 \%$ and $45.5 \%$ respectively) while $91.4 \%$ had sunscreen protection In reference to laboratory work-up and monitoring, complete laboratory work-up at diagnosis was performed in $48 \%$, while disease activity and damage, were fully assessed only in $14.1 \%$ (in three consecutive visits) and $28.6 \%$ (annually) respectively, Similarly, reproductive health and pregnancy counselling adherence rates were modest estimated at $50 \%$ and $62 \%$ respectively. Higher adherence to the indicated care during follow-up (monitoring QIs) was associated with reduced risk for adverse outcomes during the last year of observation (OR $0.97,95 \% \mathrm{Cl}$ 0.96-0.99). Patients who achieved sustained remission or LLDAS, exhibited fewer flares $(O R=0.15$, $p$-value $<0.001)$ and damage accrual $(O R=0.35$, $p$-value $<0.001)$ Of interest, patients who received low-dose of GCs or were appropriately vaccinated, had a lower risk of experiencing a flare ( $\mathrm{OR}=0.23$ and 0.46 respectively). 
Conclusion: A set of 18 Qls based on the EULAR recommendations for SLE was developed to be used towards improving care in SLE. Initial real-life data suggest variable degree of adherence with higher adherence resulting in reduced adverse outcomes. REFERENCES:

[1] Fanouriakis, et al., 2019 Update of the EULAR recommendations for the management of systemic lupus erythematosus. In Annals of the Rheumatic Diseases (Vol. 78, Issue 6, pp. 736-745). BMJ Publishing Group. https://doi. org/10.1136/annrheumdis-2019-215089.

[2] Nikolopoulos, D., et al., Evolving phenotype of systemic lupus erythematosus in Caucasians: low incidence of lupus nephritis, high burden of neuropsychiatric disease and increased rates of late-onset lupus in the 'Attikon' cohort. Lupus, 29(5), 514-522. https://doi.org/10.1177/0961203320908932.

\section{Figure 1.}

Review of the literature and EULAR 2019 Recommendations for SLE

ป

Preliminary set of 44 Qls

$\sqrt{1}$

Identification of level of evidence for each QI

I

Set of 44 Qls reviewed by a panel of experts

$\sqrt{ }$

Experts scored each item for validity and feasibility using a 9-point scale

\section{II}

Analysis of each candidate QI using the RAND/UCLA Appropriateness Method

n

Revised set of 18 Qls based on median rating, median absolute deviation, lower limit inter-percentile range (IPR), upper limit IPR, Disagreement Index (DI) and expert's comments for each QI,

\section{$\sqrt{1}$}

Second and final re-rating round of the 18 revised Qls

Acknowledgements: This project has received funding from the European Research Council (ERC) under the European Union's Horizon 2020 research and innovation programme (grant agreement No 742390)

Disclosure of Interests: None declared

DOI: 10.1136/annrheumdis-2021-eular.3181

\section{POS0765 LABORATORY RATIOS: A SUBROGATE BIOMARKER FOR DETECTION OF INFECTION IN SLE PATIENTS}

Y. Santamaria-Alza ${ }^{1}$, J. Sanchez-Bautista ${ }^{2}$, T. Urrego Callejas ${ }^{1}$, J. Moreno ${ }^{2}$, F. Jaimes ${ }^{2}$, J. Rodello ${ }^{3}$, D. Fernández ${ }^{4}$, L. A. González ${ }^{1}$, G. Vásquez ${ }^{1}$.

${ }^{1}$ Universidad de Antioquia, Rheumatology, Medellín, Colombia; ${ }^{2}$ Universidad de Antioquia, Internal Medicine, Medellín, Colombia; ${ }^{3}$ Universidad de Antioquia, Nephrology, Medellín, Colombia; ${ }^{4}$ Polytechnic University of CataloniaBarcelonaTech, Statistics and Operations Research, Medellín, Colombia

Background: The most common complication in patients with SLE is infection, and its clinical presentation is often indistinguishable from SLE flares. Therefore, laboratory ratios have been evaluated to differentiate between those events Among them, ESR/CRP ${ }^{1}$, neutrophil/lymphocyte $(\mathrm{NLR})^{2}$, and platelet/lymphocyte $(P L R)^{3}$ ratios have been previously assessed with acceptable performance; how ever, there is no validation of those ratios in our SLE population.
Objectives: To examine the predictive capacity of infection of the lymphocyte/C4 (LC4R), lymphocyte/C3 (LC3R), and ferritin/ESR (FER) ratios in SLE patients, and to evaluate the performance of ESR/CRP, NLR, AND PLR ratios in our SLE population. Methods: We conducted a cross-sectional study of SLE patients admitted to the emergency service at Hospital San Vicente Fundación (HSVF). The HSVF ethics committee approved the execution of the project.

Patients were categorized into four groups according to the main cause of hospitalization: (1) infection, (2) flare, (3) infection and flare and, (4) neither infection nor flare. We calculated the median values of the ratios and their respective interquartile ranges for each group. Then, we compared those summary measures using the Kruskal-Wallis test. Subsequently, we assessed the predictive capacity of infection of each ratio using ROC curve. Finally, we carried out a logistic regression model. Results: A total of 246 patients were included, among them $90.7 \%$ were women The median age was 28 years (IQR: 20-35 years). Regarding the outcomes $37.0 \%$ of the patients had flares, $30.9 \%$ had neither infection nor flare, $16.7 \%$ had an infection and, $15.5 \%$ had simultaneously infection and flare. When compared the four groups, statistical significance $(p<0.05)$ was observed. Area under the ROC curve (AUC) for infection prediction was as follows: 0.752 (sensitivity $60.5 \%$, specificity $80.5 \%$ ) for LC4R, 0.740 (sensitivity $73.2 \%$, specificity $68.3 \%$ ) for FER, 0.731 (sensitivity $77.6 \%$, specificity $80.5 \%$ ) for LC3R.

In the logistic regression modeling, we observed that an increase in the risk of infection was associated with an LC4R below 66.7 (OR: 6.3, Cl: 2.7 - 14.3, p $<0.0001$ ), a FER greater than 13.6 (OR: $5.9, \mathrm{Cl}: 2.8-12.1, \mathrm{p}<0.0001$ ) and an LC3R below 11.2 (OR: 4.9, Cl: $2.4-9.8, \mathrm{p}<0.0001$ ).

The ESR/CRP and PLR performed poorly with an AUC of 0.580 and 0.655 respectively. In contrast, the NLR showed better performance (AUC of 0.709 , with a sensitivity of $80.2 \%$ and specificity of $55.7 \%$ ).
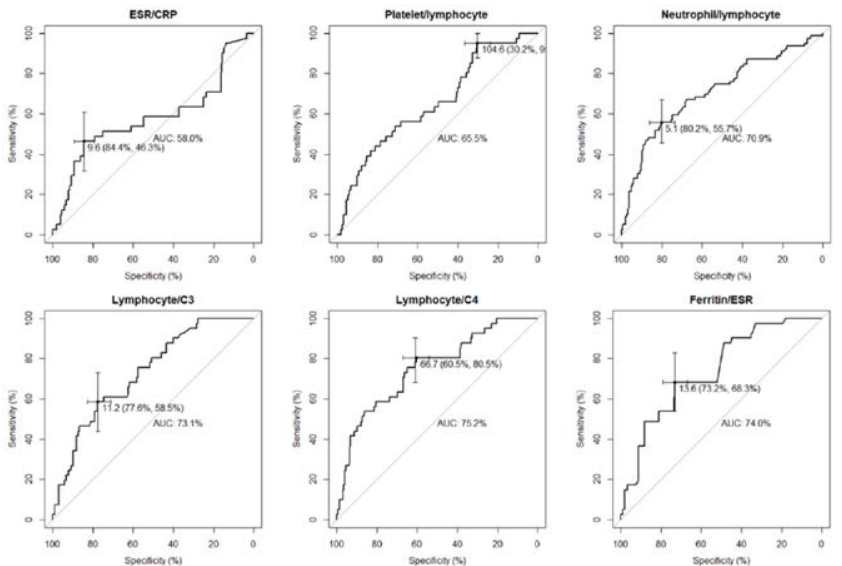

Figure 1. ROC curves of the evaluated ratios

Conclusion: These laboratory ratios could be easy to assay and inexpen sive biomarkers to differentiate between infection and activity in SLE patients. The LC4R, FER, and LC3R have a significant diagnostic performance for detecting infection among SLE patients. Of the ratios previously evaluated, ESR/CRP, LPR, NLR, only the latest has an adequate performance in our population.

\section{REFERENCES:}

[1] Littlejohn E, Marder W, Lewis E, et al. The ratio of erythrocyte sedimentation rate to $\mathrm{C}$-reactive protein is useful in distinguishing infection from flare in systemic lupus erythematosus patients presenting with fever. Lupus. 2018;27(7):1123-1129.

[2] Broca-Garcia BE, Saavedra MA, Martínez-Bencomo MA, et al. Utility of neutrophil-to-lymphocyte ratio plus C-reactive protein for infection in systemic lupus erythematosus. Lupus. 2019;28(2):217-222.

[3] Soliman WM, Sherif NM, Ghanima IM, EL-Badawy MA. Neutrophil to lymphocyte and platelet to lymphocyte ratios in systemic lupus erythematosus: Relation with disease activity and lupus nephritis. Reumatol Clin. 2020;16(4):255-261s

Disclosure of Interests: None declared

DOI: 10.1136/annrheumdis-2021-eular.3184

\section{POS0766 CLUSTER ANALYSIS AND COMPARISON OF CUMULATIVE DAMAGE BY DIAPS IN A SINGLE CENTER COHORT OF APS PATIENTS}

O. Uludag ${ }^{1}$, E. Gurel ${ }^{1}$, C. Cetin ${ }^{1}$, E. Cene ${ }^{2}$, Y. Yalçınkaya ${ }^{1}$, A. Gül ${ }^{1}$, M. Inanc ${ }^{1}$, B. Artim-Esen ${ }^{1}$. ${ }^{1}$ Istanbul Faculty of Medicine, Division of Rheumatology, Department of Internal Medicine, İstanbul, Turkey; ${ }^{2}$ Faculty of Arts and Science, Yıldız Technical University, Department of Statistics, Istanbul, Turkey 